\title{
Evaluation of the effects of an interdisciplinary lifestyle and health program for adolescents and its benefits for maintaining health.
}

Anja Schuster ( $\square$ anja.schuster@fh-salzburg.ac.at)

Fachhochschule Salzburg https://orcid.org/0000-0003-2712-9245

Karin Schwenoha

Fachhochschule Salzburg GmbH

Edith Oberkofler

HBLA Ursprung

Gerald Lirk

University of Applied Sciences Upper Austria

Elisabeth Ardelt-Gattinger

Universitat Salzburg

Daniel Weghuber

Paracelsus Medizinische Privatuniversitat

\section{Susanne Ring-Dimitriou}

Universitat Salzburg

Thomas Freudenthaler

Universitat Salzburg

Gertie J. Oostingh

Fachhochschule Salzburg

Barbara Bogner

Paracelsus Medizinische Privatuniversitat

\section{Research}

Keywords: anthropometric data, biomedical parameters, fitness evaluation, AD-EVA questionnaire, physical fitness test battery, adolescents

Posted Date: August 3rd, 2020

DOI: https://doi.org/10.21203/rs.3.rs-44118/v2

License: (a) (i) This work is licensed under a Creative Commons Attribution 4.0 International License.

Read Full License 

The authors have withdrawn this preprint from Research Square 